\title{
Transmission, Intravenous Drug Abuser
}

National Cancer Institute

\section{Source}

National Cancer Institute. Transmission, Intravenous Drug Abuser. NCI Thesaurus. Code C19081.

Passage or transfer, as of a disease, by intravenous injection with shared drug paraphernalia from a donor intravenous drug abuser to a recipient intravenous drug abuser. $(\mathrm{NCl})$ 